\title{
Improved Integral Inequalities for Stability Analysis of Interval Time-Delay Systems
}

\author{
Shuai Zhang * (1) and Xiao Qi \\ School of Control and Computer Engineering, North China Electric Power University, Beijing 102206, China; \\ qixiao@ncepu.edu.cn \\ * Correspondence: zhangshuaixufeng@163.com; Tel.: +86-188-1034-8278
}

Received: 28 October 2017; Accepted: 30 November 2017; Published: 3 December 2017

\begin{abstract}
A novel stability analysis for the interval time-delay systems is proposed by employing a new series of integral inequalities for single and double integrals. Different from the recently introduced Wirtinger-based inequalities, refined Jensen inequalities and auxiliary function-based inequalities, the proposed ones can provide more accurate bounds for the cross terms in derivatives of the Lyapunov-Krasovskii functional (LKF) without involving additional slack variables. Based on the augmented LKF with triple-integral terms, their applications to stability analysis for interval time-delay systems are provided. By virtue of the newly derived inequalities, the resulting criteria are less conservative than some existing literature. Finally, numerical examples are provided to verify the effectiveness and improvement of the proposed approaches.
\end{abstract}

Keywords: improved integral inequalities; time-delay systems; interval time-varying delays; stability

\section{Introduction}

Because of the finite speed of data transmission, time delays are unavoidably encountered in a variety of real-world systems, such as multi-agent systems [1,2], active suspension systems [3,4], chemical engineering systems [5], and so on. The time delays frequently cause undesirable dynamic behaviors [6,7]. Consequently, a considerable amount of attention has been paid to the stability analysis for time-delay systems [8-15].

In this area, the Lyapunov-Krasovskii functional (LKF) is the most efficient mathematical tool $[4,5]$. It is the main idea of the LKF based approach to establish a positive definite functional such that its derivative along a solution of the considered system is a definite negative [7]. However, a certain degree of conservatism is inevitably introduced, since only sufficient conditions can be obtained. As is well known, the reduction of conservatism depends on the construction of LKF to a considerable extent, which aims at making use of more information about the delay [11]. In this trend, a great deal of effort has been contributed, including augmented LKF [11], delay decomposition/fractioning [12,13], triple-integral terms $[14,15]$ and functional including quadratic terms multiplied by a higher degree scalar function [16].

The reduction of conservatism also benefits from the techniques utilized to estimate the cross terms when differentiating the LKF [17]. Compared with the choice of LKF, the accurate bounding technique has been considered as a more effective manner to relax the criteria. In this regard, most of the contributions are derived via Jensen inequality thanks to its convenient tractability [13-15]. However, as discussed in [18], the Jensen inequality often leads to undesirable conservatism. Thus, reducing the estimation gap of the Jensen inequality has become an open issue. Recently, an alternative inequality based on the Wirtinger inequality has been proposed in [19] to achieve a potential gain with respect to Jensen inequality. In [20], by using information on the double integral of state, a new free-matrix-based integral inequality is developed, which includes the Wirtinger one at the cost of computational burden [21]. 
In [17], a novel modification method is introduced to derive a new integral inequality. If the LKF with triple-integral terms are established, the search for how to provide tighter bounds for double integral functionals is becoming a stringent task. In [22], the Wirtinger-based inequality is extended to the double integral form. In [23], the Jensen-based inequalities are refined for both single and double integrals and their potential capacity exhibits obvious advantages over the previous ones. In [24], by using Wirtinger-based single and double integral inequalities and delay decomposition technique, the stability analysis of neural systems is investigated. Combining advanced integral inequalities and slack variables, a new delay-dependent stability condition for time delay systems is developed in [25]. Among the literature on this subject, the most noticeable technique is the auxiliary function-based integral inequalities performed in [26], which are suitable for the LKF in triple integral forms and encompass the Jensen and Wirtinger ones as special cases. Therefore, how to develop new integral inequalities to provide more accurate bounds for both single and double integrals than [26] motivates the present study.

Based on the above discussions, the main contribution of this paper is that a novel series of integral inequalities independent of slack variables is proposed, which shows significant improvements over the Wirtinger-based, refined Jensen and auxiliary function-based ones. By constructing an augmented LKF with triple-integral terms, the information on the delay range, especially on the lower bound of delay, is fully taken into consideration. By virtue of the improved inequalities, the derived stability conditions are less conservative than some existing ones.

The remainder of the current paper is organized as follows: the problem formulation and preliminary are presented in Section 2. Section 3 provides the new integral inequalities. In Section 4 , the stability criteria for interval time-delay systems is obtained. Numerical examples are presented in Section 5 to demonstrate the effectiveness of the proposed approaches. Section 6 draws the conclusions.

Notation: In this paper, $\Re^{n}$ is the $n$-dimensional Euclidean space and $\Re^{n \times m}$ is the set of all $n \times m$ real matrices. * refers to the symmetric term in a symmetric matrix. The superscripts $T$ and -1 are the transpose and inverse of a matrix, respectively. The notation $P>0(P \geq 0)$ means that the matrix $P$ is symmetric positive definite (positive semi-definite). $I$ and 0 stand for the identity and zero matrix,

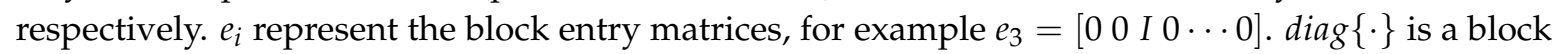
diagonal matrix, $\operatorname{col}\{\cdot\}$ means a column vector, and $\operatorname{sym}\{P\}=P+P^{T}$.

\section{Problem Statement and Preliminary}

Consider the following linear system with an interval time-varying delay:

$$
\left\{\begin{array}{l}
\dot{x}(t)=A x(t)+B x(t-d(t)), t \geq 0 \\
x(t)=\phi(t), t \in\left[-d_{2}, 0\right],
\end{array}\right.
$$

where $x(t) \in \Re^{n}$ is the system state, $A, B \in \Re^{n \times n}$ are constant system matrices, the initial condition $\phi(t)$ is a continuously differentiable function on $\left[-d_{2}, 0\right]$ and $d(t)$ is an interval time delay satisfying:

$$
d(t) \in\left[d_{1}, d_{2}\right], \dot{d}(t) \in[0, \mu]
$$

where $d_{2}>d_{1}>0$ and $\mu>0$ are constants. Next, the reciprocally convex lemma is presented, which will be useful in deriving the criteria.

Lemma 1. ([27]) Let $f_{1}, f_{2}, \cdots, f_{N}: \Re^{m} \mapsto \Re$ have positive values in open subset $D$ of $\Re^{m}$. The reciprocally convex combination of $f_{i}$ over $D$ satisfies

$$
\min _{\left\{\alpha_{i} \mid \alpha_{i}>0, \sum_{i} \alpha_{i}=1\right\}} \sum_{i} \frac{1}{\alpha_{i}} f_{i}(t)=\sum_{i} f_{i}(t)+\max _{g_{i, j}(t)} \sum_{i \neq j} g_{i, j}(t),
$$

subject to 


$$
\left\{g_{i, j}: \Re^{m} \mapsto \Re, g_{j, i}(t)=g_{i, j}(t),\left[\begin{array}{cc}
f_{i}(t) & g_{i, j}(t) \\
g_{i, j}(t) & f_{j}(t)
\end{array}\right] \geq 0\right\} .
$$

\section{New Integral Inequalities}

As discussed in the first section, the reduction of conservatism primarily benefits from two factors: the suitable construction of LKF and more accurate estimation for its derivative. Revisiting the literature $[14,17,23,26]$, the most commonly used LKF consists of the following double and triple integral terms:

$$
\begin{gathered}
V_{D}(t)=\int_{t-d_{2}}^{t-d_{1}} \int_{\theta}^{t} \dot{x}^{T}(s) Q_{1} \dot{x}(s) d s d \theta \\
V_{T}(t)=\int_{t-d_{2}}^{t-d_{1}} \int_{\sigma}^{t-d_{1}} \int_{\theta}^{t} \dot{x}^{T}(s) Q_{2} \dot{x}(s) d s d \theta d \sigma+\int_{t-d_{2}}^{t-d_{1}} \int_{t-d_{2}}^{\sigma} \int_{\theta}^{t} \dot{x}^{T}(s) Q_{3} \dot{x}(s) d s d \theta d \sigma,
\end{gathered}
$$

where $Q_{i}>0(i=1,2,3)$ are the Lyapunov matrices. The derivatives of $V_{D}(t)$ and $V_{T}(t)$ are given by

$$
\begin{gathered}
\dot{V}_{D}(t)=\left(d_{2}-d_{1}\right) \dot{x}^{T}(t) Q_{1} \dot{x}(t)-\int_{t-d_{2}}^{t-d_{1}} \dot{x}^{T}(s) Q_{1} \dot{x}(s) d s \\
\dot{V}_{T}(t)=\frac{\left(d_{2}-d_{1}\right)^{2}}{2} \dot{x}^{T}(t)\left(Q_{2}+Q_{3}\right) \dot{x}(t)-\int_{t-d_{2}}^{t-d_{1}} \int_{\theta}^{t-d_{1}} \dot{x}^{T}(s) Q_{2} \dot{x}(s) d s d \theta-\int_{t-d_{2}}^{t-d_{1}} \int_{t-d_{2}}^{\theta} \dot{x}^{T}(s) Q_{3} \dot{x}(s) d s d \theta
\end{gathered}
$$

In order to obtain a linear matrix inequality (LMI)-based stability condition and less conservatism, it is required to provide closer estimations for the integrals of quadratic functions in Equations (7) and (8). For this purpose, a new set of integral inequalities is developed in the following lemmas.

Lemma 2. For a given matrix $Q>0$, the following inequality holds for all continuously differentiable function $x$ in $[\alpha, \beta] \rightarrow \Re^{n}$ :

$$
\int_{\alpha}^{\beta} \dot{x}^{T}(s) Q \dot{x}(s) d s \geq \frac{1}{\beta-\alpha}\left(\psi_{1}^{T} Q \psi_{1}+3 \psi_{2}^{T} Q \psi_{2}+5 \psi_{3}^{T} Q \psi_{3}+7 \psi_{4}^{T} Q \psi_{4}\right),
$$

where

$$
\begin{gathered}
\psi_{1}=x(\beta)-x(\alpha), \psi_{2}=x(\beta)+x(\alpha)-\frac{2}{\beta-\alpha} \int_{\alpha}^{\beta} x(s) d s, \\
\psi_{3}=x(\beta)-x(\alpha)+\frac{6}{\beta-\alpha} \int_{\alpha}^{\beta} x(s) d s-\frac{12}{(\beta-\alpha)^{2}} \int_{\alpha}^{\beta} \int_{\theta}^{\beta} x(s) d s d \theta \\
\psi_{4}=x(\beta)+x(\alpha)-\frac{12}{\beta-\alpha} \int_{\alpha}^{\beta} x(s) d s+\frac{60}{(\beta-\alpha)^{2}} \int_{\alpha}^{\beta} \int_{\theta}^{\beta} x(s) d s d \theta-\frac{120}{(\beta-\alpha)^{3}} \int_{\alpha}^{\beta} \int_{\sigma}^{\beta} \int_{\theta}^{\beta} x(s) d s d \theta d \sigma .
\end{gathered}
$$

Proof. For any differentiable function $x$, define a function $\omega_{1}(s)$ for all $s \in[\alpha, \beta]$ given by:

$$
\omega_{1}(s)=\dot{x}(s)-\frac{1}{\beta-\alpha} \int_{\alpha}^{\beta} \dot{x}(s) d s-\delta_{1}(s) \vartheta_{1}-\delta_{2}(s) \vartheta_{2}-\delta_{3}(s) \vartheta_{3}
$$

where $\vartheta_{i}(i=1,2,3)$ are constant vectors to be determined and

$$
\begin{gathered}
\delta_{1}(s)=\frac{2 s-\alpha-\beta}{2}, \delta_{2}(s)=\frac{12 s^{2}-12(\alpha+\beta) s+2 \alpha^{2}+2 \beta^{2}+8 \alpha \beta}{12}, \\
\delta_{3}(s)=\frac{40 s^{3}-60(\alpha+\beta) s^{2}+24\left(\alpha^{2}+\beta^{2}+3 \alpha \beta\right) s-2 \alpha^{3}-2 \beta^{3}-9 \alpha^{2} \beta-9 \alpha \beta^{2}}{40} .
\end{gathered}
$$

For a matrix $Q>0$, integrating $\omega_{1}^{T}(s) Q \omega_{1}(s)$ from $\alpha$ to $\beta$ leads to 


$$
\begin{gathered}
\int_{\alpha}^{\beta} \omega_{1}^{T}(s) Q \omega_{1}(s) d s=\int_{\alpha}^{\beta} \dot{x}^{T}(s) Q \dot{x}(s) d s-\frac{1}{\beta-\alpha} \int_{\alpha}^{\beta} \dot{x}^{T}(s) d s Q \int_{\alpha}^{\beta} \dot{x}(s) d s-2 \sum_{i=1}^{3} \int_{\alpha}^{\beta} \dot{x}^{T}(s) Q \delta_{i}(s) d s \vartheta_{i} \\
+\frac{2}{\beta-\alpha} \int_{\alpha}^{\beta} \dot{x}^{T}(s) d s Q \sum_{i=1}^{3} \int_{\alpha}^{\beta} \delta_{i}(s) d s \vartheta_{i}+\sum_{i=1}^{3} \int_{\alpha}^{\beta} \delta_{i}^{2}(s) d s \vartheta_{i}^{T} Q \vartheta_{i}+2 \int_{\alpha}^{\beta} \delta_{1}(s) \delta_{2}(s) d s \vartheta_{1}^{T} Q \vartheta_{2} \\
+2 \int_{\alpha}^{\beta} \delta_{1}(s) \delta_{3}(s) d s \vartheta_{1}^{T} Q \vartheta_{3}+2 \int_{\alpha}^{\beta} \delta_{2}(s) \delta_{3}(s) d s \vartheta_{2}^{T} Q \vartheta_{3} \geq 0 .
\end{gathered}
$$

By noting $\int_{\alpha}^{\beta} \delta_{i}(s) d s=0(i=1,2,3), \int_{\alpha}^{\beta} \delta_{i}(s) \delta_{j}(s) d s=0(i=1,2,3 ; j=1,2,3 ; i \neq j)$, it yields

$$
\int_{\alpha}^{\beta} \dot{x}^{T}(s) Q \dot{x}(s) d s \geq \frac{1}{\beta-\alpha} \int_{\alpha}^{\beta} \dot{x}^{T}(s) d s Q \int_{\alpha}^{\beta} \dot{x}(s) d s+\sum_{i=1}^{3}\left(2 \int_{\alpha}^{\beta} \dot{x}^{T}(s) Q \delta_{i}(s) d s \vartheta_{i}-\int_{\alpha}^{\beta} \delta_{i}^{2}(s) d s \vartheta_{i}^{T} Q \vartheta_{i}\right) .
$$

Rewriting the last term of the right-hand side of Equation (12) as sums of squares, one has

$$
\begin{aligned}
\int_{\alpha}^{\beta} \dot{x}^{T}(s) Q \dot{x}(s) d s & \geq \frac{1}{\beta-\alpha} \int_{\alpha}^{\beta} \dot{x}^{T}(s) d s Q \int_{\alpha}^{\beta} \dot{x}(s) d s+\sum_{i=1}^{3}\left(\int_{\alpha}^{\beta} \delta_{i}^{2}(s) d s\right)^{-1}\left(\int_{\alpha}^{\beta} \delta_{i}(s) \dot{x}(s) d s\right)^{T} Q\left(\int_{\alpha}^{\beta} \delta_{i}(s) \dot{x}(s) d s\right) \\
& -\sum_{i=1}^{3} \int_{\alpha}^{\beta} \delta_{i}^{2}(s) d s\left(\vartheta_{i}-\lambda_{i}\right)^{T} Q\left(\vartheta_{i}-\lambda_{i}\right) .
\end{aligned}
$$

where $\lambda_{i}=\left(\int_{\alpha}^{\beta} \delta_{i}^{2}(s) d s\right)^{-1} \int_{\alpha}^{\beta} \delta_{i}(s) \dot{x}(s) d s(i=1,2,3)$.

Equation (13) holds independently of the choice of constant vectors $\vartheta_{i}(i=1,2,3)$ and the last term of the right-hand side of Equation (13) is non-positive. Therefore, choosing $\vartheta_{i}=\lambda_{i}(i=1,2,3)$ leads to the maximum of the right-hand side of Equation (13). Rearranging Equation (13) leads to Equation (9). Thus, the proof is completed.

Remark 1. By the Wirtinger-based inequality [19], the new inequality [17] and the auxiliary function-based inequality [26] (the refined Jensen inequality [23]), respectively, $\int_{\alpha}^{\beta} \dot{x}^{T}(s) Q \dot{x}(s) d s$ can be estimated as follows:

$$
\begin{gathered}
\int_{\alpha}^{\beta} \dot{x}^{T}(s) Q \dot{x}(s) d s \geq \frac{1}{\beta-\alpha}\left(\psi_{1}^{T} Q \psi_{1}+3 \psi_{2}^{T} Q \psi_{2}\right), \\
\int_{\alpha}^{\beta} \dot{x}^{T}(s) Q \dot{x}(s) d s \geq \frac{1}{\beta-\alpha}\left(\psi_{1}^{T} Q \psi_{1}+5 \psi_{3}^{T} Q \psi_{3}\right), \\
\int_{\alpha}^{\beta} \dot{x}^{T}(s) Q \dot{x}(s) d s \geq \frac{1}{\beta-\alpha}\left(\psi_{1}^{T} Q \psi_{1}+3 \psi_{2}^{T} Q \psi_{2}+5 \psi_{3}^{T} Q \psi_{3}\right),
\end{gathered}
$$

Compared with Equation (14)-(16), one can see that Equation (9) produces a more accurate bound for a single integral term. Thus, the stability criteria by Lemma 2 tend to be less conservative. Unlike the existing ones, not only the relations between $x(\beta), x(\alpha), \int_{\alpha}^{\beta} x(s) d s, \int_{\alpha}^{\beta} \int_{\theta}^{\beta} x(s) d s d \theta$, but also between them and $\int_{\alpha}^{\beta} \int_{\sigma}^{\beta} \int_{\theta}^{\beta} x(s) d s d \theta d \sigma$ are all taken into account, which allows one to make use of extra information on time delay. Moreover, no extra slack matrices are introduced in Equation (9).

Lemma 3. For a given matrix $Q>0$, the following inequalities hold for all continuously differentiable function $x$ in $[\alpha, \beta] \rightarrow \Re^{n}$ :

$$
\begin{aligned}
& \int_{\alpha}^{\beta} \int_{\theta}^{\beta} \dot{x}^{T}(s) Q \dot{x}(s) d s d \theta \geq 2\left(\psi_{5}^{T} Q \psi_{5}+2 \psi_{6}^{T} Q \psi_{6}+3 \psi_{7}^{T} Q \psi_{7}\right), \\
& \int_{\alpha}^{\beta} \int_{\alpha}^{\theta} \dot{x}^{T}(s) Q \dot{x}(s) d s d \theta \geq 2\left(\psi_{8}^{T} Q \psi_{8}+2 \psi_{9}^{T} Q \psi_{9}+3 \psi_{10}^{T} Q \psi_{10}\right),
\end{aligned}
$$


where

$$
\begin{gathered}
\psi_{5}=x(\beta)-\frac{1}{\beta-\alpha} \int_{\alpha}^{\beta} x(s) d s, \psi_{6}=x(\beta)+\frac{2}{\beta-\alpha} \int_{\alpha}^{\beta} x(s) d s-\frac{6}{(\beta-\alpha)^{2}} \int_{\alpha}^{\beta} \int_{\theta}^{\beta} x(s) d s d \theta \\
\psi_{7}=x(\beta)-\frac{3}{\beta-\alpha} \int_{\alpha}^{\beta} x(s) d s+\frac{24}{(\beta-\alpha)^{2}} \int_{\alpha}^{\beta} \int_{\theta}^{\beta} x(s) d s d \theta-\frac{60}{(\beta-\alpha)^{3}} \int_{\alpha}^{\beta} \int_{\sigma}^{\beta} \int_{\theta}^{\beta} x(s) d s d \theta d \sigma \\
\psi_{8}=x(\alpha)-\frac{1}{\beta-\alpha} \int_{\alpha}^{\beta} x(s) d s, \psi_{9}=x(\alpha)-\frac{4}{\beta-\alpha} \int_{\alpha}^{\beta} x(s) d s+\frac{6}{(\beta-\alpha)^{2}} \int_{\alpha}^{\beta} \int_{\theta}^{\beta} x(s) d s d \theta \\
\psi_{10}=x(\alpha)-\frac{9}{\beta-\alpha} \int_{\alpha}^{\beta} x(s) d s+\frac{36}{(\beta-\alpha)^{2}} \int_{\alpha}^{\beta} \int_{\theta}^{\beta} x(s) d s d \theta-\frac{60}{(\beta-\alpha)^{3}} \int_{\alpha}^{\beta} \int_{\sigma}^{\beta} \int_{\theta}^{\beta} x(s) d s d \theta d \sigma .
\end{gathered}
$$

Proof. In order to prove Equation (17), consider a function $\omega_{2}(s)$ for all $s \in[\alpha, \beta]$ defined as

$$
\omega_{2}(s)=\dot{x}(s)-\frac{2}{(\beta-\alpha)^{2}} \int_{\alpha}^{\beta} \int_{\theta}^{\beta} \dot{x}(s) d s d \theta-\delta_{4}(s) \vartheta_{4}-\delta_{5}(s) \vartheta_{5},
$$

where $\vartheta_{4}$ and $\vartheta_{5}$ are constant vectors to be determined and

$$
\delta_{4}(s)=\frac{-3 s+\alpha+2 \beta}{3}, \delta_{5}(s)=\frac{10 s^{2}-8 \alpha s-12 \beta s+\alpha^{2}+3 \beta^{2}+6 \alpha \beta}{10},
$$

satisfying $\int_{\alpha}^{\beta} \int_{\theta}^{\beta} \delta_{4}(s) d s d \theta=0, \int_{\alpha}^{\beta} \int_{\theta}^{\beta} \delta_{5}(s) d s d \theta=0$ and $\int_{\alpha}^{\beta} \int_{\theta}^{\beta} \delta_{4}(s) \delta_{5}(s) d s d \theta=0$.

For a matrix $Q>0$, one has

$$
\begin{aligned}
\int_{\alpha}^{\beta} \int_{\theta}^{\beta} \omega_{2}^{T}(s) Q \omega_{2}(s) d s d \theta & =\int_{\alpha}^{\beta} \int_{\theta}^{\beta} \dot{x}^{T}(s) Q \dot{x}(s) d s d \theta-\frac{2}{(\beta-\alpha)^{2}} \int_{\alpha}^{\beta} \int_{\theta}^{\beta} \dot{x}^{T}(s) d s d \theta Q \int_{\alpha}^{\beta} \int_{\theta}^{\beta} \dot{x}(s) d s d \theta \\
& -2 \sum_{i=4}^{5} \int_{\alpha}^{\beta} \int_{\theta}^{\beta} \dot{x}^{T}(s) Q \delta_{i}(s) d s d \theta \vartheta_{i}+\sum_{i=4}^{5} \int_{\alpha}^{\beta} \int_{\theta}^{\beta} \delta_{i}^{2}(s) d s d \theta \vartheta_{i}^{T} Q \vartheta_{i} \geq 0
\end{aligned}
$$

From Equation (20), one can obtain

$$
\begin{aligned}
& \int_{\alpha}^{\beta} \int_{\theta}^{\beta} \dot{x}^{T}(s) Q \dot{x}(s) d s d \theta \geq \frac{2}{(\beta-\alpha)^{2}} \int_{\alpha}^{\beta} \int_{\theta}^{\beta} \dot{x}^{T}(s) d s d \theta Q \int_{\alpha}^{\beta} \int_{\theta}^{\beta} \dot{x}(s) d s d \theta+\sum_{i=4}^{5}\left(\int_{\alpha}^{\beta} \int_{\theta}^{\beta} \delta_{i}^{2}(s) d s d \theta\right)^{-1} \\
& \times\left(\int_{\alpha}^{\beta} \int_{\theta}^{\beta} \delta_{i}(s) \dot{x}(s) d s d \theta\right)^{T} Q\left(\int_{\alpha}^{\beta} \int_{\theta}^{\beta} \delta_{i}(s) \dot{x}(s) d s d \theta\right)-\sum_{i=4}^{5} \int_{\alpha}^{\beta} \int_{\theta}^{\beta} \delta_{i}^{2}(s) d s d \theta\left(\vartheta_{i}-\lambda_{i}\right)^{T} Q\left(\vartheta_{i}-\lambda_{i}\right) .
\end{aligned}
$$

where $\lambda_{i}=\left(\int_{\alpha}^{\beta} \int_{\theta}^{\beta} \delta_{i}^{2}(s) d s d \theta\right)^{-1} \int_{\alpha}^{\beta} \int_{\theta}^{\beta} \delta_{i}(s) \dot{x}(s) d s d \theta(i=4,5)$.

By setting $\vartheta_{i}=\lambda_{i}(i=4,5)$ to rearrange Equation (21), one can obtain Equation (17). On the other hand, choose

$$
\omega_{3}(s)=\dot{x}(s)-\frac{2}{(\beta-\alpha)^{2}} \int_{\alpha}^{\beta} \int_{\alpha}^{\theta} \dot{x}(s) d s d \theta-\delta_{6}(s) \vartheta_{6}-\delta_{7}(s) \vartheta_{7},
$$

where $\vartheta_{6}$ and $\vartheta_{7}$ are constant vectors to be determined and $\delta_{6}(s)=\frac{3 s-2 \alpha+\beta}{3}$, $\delta_{7}(s)=\frac{10 s^{2}-12 \alpha s-8 \beta s+3 \alpha^{2}+\beta^{2}+6 \alpha \beta}{10}$. By proceeding the proof process similar to that of Equations (17) and (18) is derived. This completes the proof.

Remark 2. By the double-integral form of Wirtinger-based inequality [22] and the auxiliary function-based inequality [26], respectively, $\int_{\alpha}^{\beta} \int_{\theta}^{\beta} \dot{x}^{T}(s) Q \dot{x}(s) d s d \theta$ can be bounded in the following two forms:

$$
\begin{gathered}
\int_{\alpha}^{\beta} \int_{\theta}^{\beta} \dot{x}^{T}(s) Q \dot{x}(s) d s d \theta \geq 2 \psi_{5}^{T} Q \psi_{5}+\psi_{6}^{T} Q \psi_{6}, \\
\int_{\alpha}^{\beta} \int_{\theta}^{\beta} \dot{x}^{T}(s) Q \dot{x}(s) d s d \theta \geq 2\left(\psi_{5}^{T} Q \psi_{5}+2 \psi_{6}^{T} Q \psi_{6}\right) .
\end{gathered}
$$


It is obvious that the proposed inequality Equation (17) achieves the desirable effect in reducing the estimation gaps of Equations (23) and (24). Furthermore, the additional signal of triple integral is utilized in Lemma 3, which could offer more information in the criteria and thus yield better performance. Then, the combination of Lemmas 2 and 3 is suitable for the LKF consisting of functionals in single, double and triple integral forms.

\section{Stability Analysis Criteria for Interval Time-Delay Systems}

In this section, in order to demonstrate the merits of the new integral inequalities, the proposed ones are applied to the stability analysis of the interval time-delay systems. For simplicity of presentation, for a matrix $Q$, define $\varphi_{1}(Q)=\operatorname{diag}\{Q 3 Q 5 Q 7 Q\}$ and $\varphi_{2}(Q)=\operatorname{diag}\{2 Q 4 Q 6 Q\}$.

Theorem 1. Given scalars $d_{2}>d_{1}>0$ and $\mu>0$, the system (1) with an interval time-delay $d(t)$ subject to (2) is asymptotically stable if there exist matrices $P=\left[P_{i j}\right]_{2 \times 2}>0, R=\left[R_{i j}\right]_{2 \times 2}>0, Q_{i}>0, U_{i}>0$, $S_{i}>0, W_{i}>0(i=1,2 ; j=1,2)$ and any matrices $Z_{l}(l=1,2,3,4)$ of appropriate dimensions such that the following LMIs hold:

$$
\begin{gathered}
{\left[\begin{array}{cc}
U_{2}+W_{1} & Z_{l} \\
* & U_{2}+W_{2}
\end{array}\right]>0(l=1,2,3,4),} \\
\Pi_{i}=\operatorname{sym}\left\{\Omega_{i}^{T} P \Lambda\right\}+\sum_{j=1}^{5} \Phi_{j}+\Gamma+\sum_{l=1}^{4} \Theta_{l}<0(i=1,2),
\end{gathered}
$$

where

$$
\begin{aligned}
& \Omega_{1}=\left[\begin{array}{c}
e_{1} \\
d e_{5}
\end{array}\right], \Omega_{2}=\left[\begin{array}{c}
e_{1} \\
d e_{6}
\end{array}\right], \Phi_{1}=\left[\begin{array}{c}
e_{1} \\
e_{4}
\end{array}\right]^{T}\left[\begin{array}{cc}
Q_{1} & 0 \\
* & -(1-\mu) Q_{1}
\end{array}\right]\left[\begin{array}{l}
e_{1} \\
e_{4}
\end{array}\right]+\left[\begin{array}{c}
e_{2} \\
e_{3}
\end{array}\right]^{T}\left[\begin{array}{cc}
Q_{2} & 0 \\
* & -Q_{2}
\end{array}\right]\left[\begin{array}{l}
e_{2} \\
e_{3}
\end{array}\right], \\
& \Lambda=\left[\begin{array}{c}
A e_{1}+B e_{4} \\
e_{2}-e_{3}
\end{array}\right], \Phi_{2}=\frac{1}{2} \operatorname{sym}\left\{\left[\begin{array}{c}
2 d_{1}\left(e_{1}-e_{7}\right) \\
d_{1}^{2}\left(e_{1}-2 e_{10}\right)
\end{array}\right]^{T}\left[\begin{array}{cc}
R_{11} & R_{12} \\
* & R_{22}
\end{array}\right]\left[\begin{array}{l}
d_{1}^{2} e_{10} \\
d_{1}^{3} e_{13}
\end{array}\right]\right\}, \\
& \Phi_{3}=\left(A e_{1}+B e_{4}\right)^{T}\left(d_{1}^{2} U_{1}+d^{2} U_{2}\right)\left(A e_{1}+B e_{4}\right), \Phi_{4}=\left(d_{1}^{2} / 2\right)\left(A e_{1}+B e_{4}\right)^{T}\left(S_{1}+S_{2}\right)\left(A e_{1}+B e_{4}\right), \\
& \Phi_{5}=\left(d^{2} / 2\right)\left(A e_{1}+B e_{4}\right)^{T}\left(W_{1}+W_{2}\right)\left(A e_{1}+B e_{4}\right), \widehat{\Gamma}=\operatorname{col}\left\{\Gamma_{1}, \Gamma_{4}, \Gamma_{5}, \Gamma_{6}, \Gamma_{7}, \Gamma_{8}, \Gamma_{9}\right\}, \\
& \Gamma=-\widehat{\Gamma}^{T} \operatorname{diag}\left\{\varphi_{1}\left(U_{1}\right), \varphi_{2}\left(S_{1}\right), \varphi_{2}\left(S_{2}\right), \varphi_{2}\left(W_{1}\right), \varphi_{2}\left(W_{1}\right), \varphi_{2}\left(W_{2}\right), \varphi_{2}\left(W_{2}\right)\right\} \widehat{\Gamma}, \\
& \Gamma_{1}=\operatorname{col}\left\{e_{1}-e_{2}, e_{1}+e_{2}-2 e_{7}, e_{1}-e_{2}+6 e_{7}-12 e_{10}, e_{1}+e_{2}-12 e_{7}+60 e_{10}-120 e_{13}\right\}, \\
& \Gamma_{2}=\operatorname{col}\left\{e_{2}-e_{4}, e_{2}+e_{4}-2 e_{5}, e_{2}-e_{4}+6 e_{5}-12 e_{8}, e_{2}+e_{4}-12 e_{5}+60 e_{8}-120 e_{11}\right\}, \\
& \Gamma_{3}=\operatorname{col}\left\{e_{4}-e_{3}, e_{4}+e_{3}-2 e_{6}, e_{4}-e_{3}+6 e_{6}-12 e_{9}, e_{4}+e_{3}-12 e_{6}+60 e_{9}-120 e_{12}\right\}, \\
& \Gamma_{4}=\operatorname{col}\left\{e_{1}-e_{7}, e_{1}+2 e_{7}-6 e_{10}, e_{1}-3 e_{7}+24 e_{10}-60 e_{13}\right\}, \\
& \Gamma_{5}=\operatorname{col}\left\{e_{2}-e_{7}, e_{2}-4 e_{7}+6 e_{10}, e_{2}-9 e_{7}+36 e_{10}-60 e_{13}\right\}, \\
& \Gamma_{6}=\operatorname{col}\left\{e_{2}-e_{5}, e_{2}+2 e_{5}-6 e_{8}, e_{2}-3 e_{5}+24 e_{8}-60 e_{11}\right\}, \\
& \Gamma_{7}=\operatorname{col}\left\{e_{4}-e_{6}, e_{4}+2 e_{6}-6 e_{9}, e_{4}-3 e_{6}+24 e_{9}-60 e_{12}\right\}, \\
& \Gamma_{8}=\operatorname{col}\left\{e_{4}-e_{5}, e_{4}-4 e_{5}+6 e_{8}, e_{4}-9 e_{5}+36 e_{8}-60 e_{11}\right\}, \\
& \Gamma_{9}=\operatorname{col}\left\{e_{3}-e_{6}, e_{3}-4 e_{6}+6 e_{9}, e_{3}-9 e_{6}+36 e_{9}-60 e_{12}\right\}, \\
& \Theta_{l}=-(2 l-1)\left[\begin{array}{l}
e_{l} \Gamma_{2} \\
e_{l} \Gamma_{3}
\end{array}\right]^{T}\left[\begin{array}{cc}
U_{2} & Z_{l} \\
* & U_{2}
\end{array}\right]\left[\begin{array}{l}
e_{l} \Gamma_{2} \\
e_{l} \Gamma_{3}
\end{array}\right](l=1,2,3,4) .
\end{aligned}
$$

Proof. Consider the following LKF candidate,

$$
V(t)=V_{1}(t)+V_{2}(t)+V_{3}(t)+V_{4}(t)+V_{5}(t)+V_{6}(t),
$$

where the individual functionals are defined as follows: 


$$
\begin{aligned}
& V_{1}(t)=\left[\begin{array}{c}
x(t) \\
\int_{t-d_{2}}^{t-d_{1}} x(s) d s
\end{array}\right]^{T}\left[\begin{array}{cc}
P_{11} & P_{12} \\
* & P_{22}
\end{array}\right]\left[\begin{array}{c}
x(t) \\
\int_{t-d_{2}}^{t-d_{1}} x(s) d s
\end{array}\right], \\
& V_{2}(t)=\int_{t-d(t)}^{t} x^{T}(s) Q_{1} x(s) d s+\int_{t-d_{2}}^{t-d_{1}} x^{T}(s) Q_{2} x(s) d s, \\
& V_{3}(t)=\left[\begin{array}{c}
\int_{t-d_{1}}^{t} \int_{\theta}^{t} x(s) d s d \theta \\
\int_{t-d_{1}}^{t} \int_{\sigma}^{t} \int_{\theta}^{t} x(s) d s d \theta d \sigma
\end{array}\right]^{T}\left[\begin{array}{cc}
R_{11} & R_{12} \\
* & R_{22}
\end{array}\right]\left[\begin{array}{c}
\int_{t-d_{1}}^{t} \int_{\theta}^{t} x(s) d s d \theta \\
\int_{t-d_{1}}^{t} \int_{\sigma}^{t} \int_{\theta}^{t} x(s) d s d \theta d \sigma
\end{array}\right], \\
& V_{4}(t)=d_{1} \int_{t-d_{1}}^{t} \int_{\theta}^{t} \dot{x}^{T}(s) U_{1} \dot{x}(s) d s d \theta+d \int_{t-d_{2}}^{t-d_{1}} \int_{\theta}^{t} \dot{x}^{T}(s) U_{2} \dot{x}(s) d s d \theta, \\
& V_{5}(t)=\int_{t-d_{1}}^{t} \int_{\sigma}^{t} \int_{\theta}^{t} \dot{x}^{T}(s) S_{1} \dot{x}(s) d s d \theta d \sigma+\int_{t-d_{1}}^{t} \int_{t-d_{1}}^{\sigma} \int_{\theta}^{t} \dot{x}^{T}(s) S_{2} \dot{x}(s) d s d \theta d \sigma, \\
& V_{6}(t)=\int_{t-d_{2}}^{t-d_{1}} \int_{\sigma}^{t-d_{1}} \int_{\theta}^{t} \dot{x}^{T}(s) W_{1} \dot{x}(s) d s d \theta d \sigma+\int_{t-d_{2}}^{t-d_{1}} \int_{t-d_{2}}^{\sigma} \int_{\theta}^{t} \dot{x}^{T}(s) W_{2} \dot{x}(s) d s d \theta d \sigma .
\end{aligned}
$$

Differentiating $V(t)$ along the solution of system (1) leads to

$$
\dot{V}_{1}(t)=v^{T}(t) \operatorname{sym}\left\{\left(\frac{d(t)-d_{1}}{d} \Omega_{1}^{T}+\frac{d_{2}-d(t)}{d} \Omega_{2}^{T}\right) P \Lambda\right\} v(t),
$$

$$
\begin{aligned}
& \dot{V}_{2}(t)=x^{T}(t) Q_{1} x(t)-(1-\dot{d}(t)) x^{T}(t-d(t)) Q_{1} x(t-d(t))+x^{T}\left(t-d_{1}\right) Q_{2} x\left(t-d_{1}\right)-x^{T}\left(t-d_{2}\right) Q_{2} x\left(t-d_{2}\right) \leq v^{T}(t) \Phi_{1} v(t), \\
& \dot{V}_{3}(t)==v^{T}(t) \Phi_{2} v(t), \\
& \dot{V}_{4}(t)=v^{T}(t) \Phi_{3} v(t)-d_{1} \int_{t-d_{1}}^{t} \dot{x}^{T}(s) U_{1} \dot{x}(s) d s-d \int_{t-d(t)}^{t-d_{1}} \dot{x}^{T}(s) U_{2} \dot{x}(s) d s-d \int_{t-d_{2}}^{t-d(t)} \dot{x}^{T}(s) U_{2} \dot{x}(s) d s, \\
& \dot{V}_{5}(t)=v^{T}(t) \Phi_{4} v(t)-\int_{t-d_{1}}^{t} \int_{\theta}^{t} \dot{x}^{T}(s) S_{1} \dot{x}(s) d s d \theta-\int_{t-d_{1}}^{t} \int_{t-d_{1}}^{\theta} \dot{x}^{T}(s) S_{2} \dot{x}(s) d s d \theta, \\
& \dot{V}_{6}(t)=v^{T}(t) \Phi_{5} v(t)-\int_{t-d(t)}^{t-d_{1}} \int_{\theta}^{t-d_{1}} \dot{x}^{T}(s) W_{1} \dot{x}(s) d s d \theta-\left(d_{2}-d(t)\right) \int_{t-d(t)}^{t-d_{1}} \dot{x}^{T}(s) W_{1} \dot{x}(s) d s \\
& -\int_{t-d_{2}}^{t-d(t)} \int_{\theta}^{t-d(t)} \dot{x}^{T}(s) W_{1} \dot{x}(s) d s d \theta-\int_{t-d(t)}^{t-d_{1}} \int_{t-d(t)}^{\theta} \dot{x}^{T}(s) W_{2} \dot{x}(s) d s d \theta \\
& -\int_{t-d_{2}}^{t-d(t)} \int_{t-d_{2}}^{\theta} \dot{x}^{T}(s) W_{2} \dot{x}(s) d s d \theta-\left(d(t)-d_{1}\right) \int_{t-d_{2}}^{t-d(t)} \dot{x}^{T}(s) W_{2} \dot{x}(s) d s,
\end{aligned}
$$

where

$$
\begin{aligned}
& d=d_{2}-d_{1}, \\
& v(t)=\operatorname{col}\left\{x(t), x\left(t-d_{1}\right), x\left(t-d_{2}\right), x(t-d(t)), c_{1}, \cdots, c_{9}\right\} \\
& c_{1}=\frac{1}{d(t)-d_{1}} \int_{t-d(t)}^{t-d_{1}} x(s) d s, \\
& c_{2}=\frac{1}{d_{2}-d(t)} \int_{t-d_{2}}^{t-d(t)} x(s) d s, \\
& c_{3}=\frac{1}{d_{1}} \int_{t-d_{1}}^{t} x(s) d s, \\
& c_{4}=\frac{1}{\left(d(t)-d_{1}\right)^{2}} \int_{t-d(t)}^{t-d_{1}} \int_{\theta}^{t-d_{1}} x(s) d s d \theta, \\
& c_{5}=\frac{1}{\left(d_{2}-d(t)\right)^{2}} \int_{t-d_{2}}^{t-d(t)} \int_{\theta}^{t-d(t)} x(s) d s d \theta, \\
& c_{6}=\frac{1}{d_{1}^{2}} \int_{t-d_{1}}^{t} \int_{\theta}^{t} x(s) d s d \theta, \\
& c_{7}=\frac{1}{\left(d(t)-d_{1}\right)^{3}} \int_{t-d(t)}^{t-d_{1}} \int_{\sigma}^{t-d_{1}} \int_{\theta}^{t-d_{1}} x(s) d s d \theta d \sigma, \\
& c_{8}=\frac{1}{\left(d_{2}-d(t)\right)^{3}} \int_{t-d_{2}}^{t-d(t)} \int_{\sigma}^{t-d(t)} \int_{\theta}^{t-d(t)} x(s) d s d \theta d \sigma, \\
& c_{9}=\frac{1}{d_{1}^{3}} \int_{t-d_{1}}^{t} \int_{\sigma}^{t} \int_{\theta}^{t} x(s) d s d \theta d \sigma .
\end{aligned}
$$

Then, the applications of Lemmas 2 and 3 to the cross terms in Equations (31)-(33) yields 


$$
\begin{gathered}
-d_{1} \int_{t-d_{1}}^{t} \dot{x}^{T}(s) U_{1} \dot{x}(s) d s-\int_{t-d_{1}}^{t} \int_{\theta}^{t} \dot{x}^{T}(s) S_{1} \dot{x}(s) d s d \theta-\int_{t-d_{1}}^{t} \int_{t-d_{1}}^{\theta} \dot{x}^{T}(s) S_{2} \dot{x}(s) d s d \theta-\int_{t-d(t)}^{t-d_{1}} \int_{\theta}^{t-d_{1}} \dot{x}^{T}(s) W_{1} \dot{x}(s) d s d \theta \\
-\int_{t-d_{2}}^{t-d(t)} \int_{\theta}^{t-d(t)} \dot{x}^{T}(s) W_{1} \dot{x}(s) d s d \theta-\int_{t-d(t)}^{t-d}(t) \int_{t-d(t)}^{\theta} \dot{x}^{T}(s) W_{2} \dot{x}(s) d s d \theta-\int_{t-d_{2}}^{t-d(t)} \int_{t-d_{2}}^{\theta} \dot{x}^{T}(s) W_{2} \dot{x}(s) d s d \theta \leq v^{T}(t) \Gamma v(t), \\
-d \int_{t-d(t)}^{t-d_{1}} \dot{x}^{T}(s) U_{2} \dot{x}(s) d s \leq\left(-d /\left(d(t)-d_{1}\right)\right) v^{T}(t) \Gamma_{2}^{T} \varphi_{1}\left(U_{2}\right) \Gamma_{2} v(t), \\
-d \int_{t-d_{2}}^{t-d(t)} \dot{x}^{T}(s) U_{2} \dot{x}(s) d s \leq\left(-d /\left(d_{2}-d(t)\right)\right) v^{T}(t) \Gamma_{3}^{T} \varphi_{1}\left(U_{2}\right) \Gamma_{3} v(t), \\
-\left(d_{2}-d(t)\right) \int_{t-d(t)}^{t-d_{1}} \dot{x}^{T}(s) W_{1} \dot{x}(s) d s \leq\left(-\left(d_{2}-d(t)\right) /\left(d(t)-d_{1}\right)\right) v^{T}(t) \Gamma_{2}^{T} \varphi_{1}\left(W_{1}\right) \Gamma_{2} v(t), \\
-\left(d(t)-d_{1}\right) \int_{t-d_{2}}^{t-d(t)} \dot{x}^{T}(s) W_{2} \dot{x}(s) d s \leq\left(-\left(d(t)-d_{1}\right) /\left(d_{2}-d(t)\right)\right) v^{T}(t) \Gamma_{3}^{T} \varphi_{1}\left(W_{2}\right) \Gamma_{3} v(t) .
\end{gathered}
$$

By Lemma 1, if there exist matrices $Z_{l}(l=1,2,3,4)$ satisfying Equation (25), the following inequality is obtained, which is similar to the treatment in [26],

$$
\begin{aligned}
& -d \int_{t-d(t)}^{t-d_{1}} \dot{x}^{T}(s) U_{2} \dot{x}(s) d s-d \int_{t-d_{2}}^{t-d(t)} \dot{x}^{T}(s) U_{2} \dot{x}(s) d s-\left(d_{2}-d(t)\right) \int_{t-d(t)}^{t-d_{1}} \dot{x}^{T}(s) W_{1} \dot{x}(s) d s-\left(d(t)-d_{1}\right) \int_{t-d_{2}}^{t-d(t)} \dot{x}^{T}(s) W_{2} \dot{x}(s) d s \\
& \leq v^{T}(t)\left(-\frac{d}{d(t)-d_{1}}\left(\sum_{l=1}^{4}(2 l-1)\left(e_{l} \Gamma_{2}\right)^{T}\left(U_{2}+W_{1}\right)\left(e_{l} \Gamma_{2}\right)\right)+\left(\sum_{l=1}^{4}(2 l-1)\left(e_{l} \Gamma_{2}\right)^{T} W_{1}\left(e_{l} \Gamma_{2}\right)\right)\right) v(t) \\
& +v^{T}(t)\left(-\frac{d}{d_{2}-d(t)}\left(\sum_{l=1}^{4}(2 l-1)\left(e_{l} \Gamma_{3}\right)^{T}\left(U_{2}+W_{2}\right)\left(e_{l} \Gamma_{3}\right)\right)+\left(\sum_{l=1}^{4}(2 l-1)\left(e_{l} \Gamma_{3}\right)^{T} W_{2}\left(e_{l} \Gamma_{3}\right)\right)\right) v(t) \\
& =v^{T}(t)\left(\sum_{l=1}^{4} \Theta_{l}\right) v(t) .
\end{aligned}
$$

By combing Equations (27)-(39), one has

$$
\dot{V}(t) \leq v^{T}(t) \Pi v(t)
$$

where

$$
\Pi=\left(\frac{d(t)-d_{1}}{d} \Omega_{1}^{T}+\frac{d_{2}-d(t)}{d} \Omega_{2}^{T}\right) P \Lambda+\sum_{j=1}^{5} \Phi_{j}+\Gamma+\sum_{l=1}^{4} \Theta_{l} .
$$

Since $\Pi$ is convex in $d(t) \in\left[d_{1}, d_{2}\right], \Pi_{1}<0$ and $\Pi_{2}<0$ ensure $\dot{V}(t)<0$, which means that the system (1) is asymptotically stable. This completes the proof.

Remark 3. By establishing a novel LKF, a stability criterion is derived in terms of LMIs. Various functionals in triple integral forms are introduced in the proposed LKF, which is effective in improving the feasible stability region [14]. Unlike the existing literatures [14,20-23,26], $\int_{t-d_{2}}^{t-d_{1}} x(s) d s, \int_{t-d_{1}}^{t} \int_{\theta}^{t} x(s) d s d \theta$ and $\int_{t-d_{1}}^{t} \int_{\sigma}^{t} \int_{\theta}^{t} x(s) d s d \theta d \sigma$ are utilized as elements of different augmented vectors such that the information on the lower bound of delay is fully exploited. Thus, the derived criterion could result in less conservatism, especially for the systems with larger lower bounds of delay.

Remark 4. More accurate bounds for the cross terms are obtained by the improved integral inequalities. As a result, it can be expected that the resulting condition has the potential to achieve more desirable performance. Unlike [14,15], handling the double integrals as a whole, more of a relationship between time-varying delay and delay range is taken into account by elaborately dividing the derivatives of triple integrals into several parts in Equation (33). By the Lemma 3, triple integrals of the state are obtained in the final result to provide more flexibility in finding the solutions of matrix variables than the approaches in $[19,20,22,23,26]$. Consequently, a great many of cross terms among double and triple integrals are obtained in the criterion, which are conducive to reducing the conservatism. 
Remark 5. Applying Lemma 3 to double integrals divided as shown in Equation (33) results in the functions weighted by $\left(d(t)-d_{2}\right) /\left(d(t)-d_{1}\right)$ and $\left(d(t)-d_{1}\right) /\left(d(t)-d_{2}\right)$. In [28], they are enlarged as $\left(d(t)-d_{2}\right) / d$ and $\left(d_{1}-d(t)\right) / d$, respectively. In Theorem 1 , using $\left(d_{2}-d(t)\right) /\left(d(t)-d_{1}\right)=$ $d /\left(d(t)-d_{1}\right)-1,\left(d(t)-d_{1}\right) /\left(d_{2}-d(t)\right)=d /\left(d_{2}-d(t)\right)-1$, the above terms and the terms with inverses of convex parameters are handled as a combination, avoiding direct approximation (see Equation (39)), which is similar to the treatment in [26]. The advantage of Theorem 1 over [26] is the substituting the Auxiliary-function based inequality by the Lemma 2. On the other hand, when the information on the delay derivative is unavailable, by setting $Q_{1}=0$, one can easily arrive at the following corollary.

Corollary 1. For given scalars $d_{2}>d_{1}>0$, the system (1) with an interval time-varying delay $d_{1} \leq d(t) \leq d_{2}$ is asymptotically stable if there exist matrices $P=\left[P_{i j}\right]_{2 \times 2}>0, R=\left[R_{i j}\right]_{2 \times 2}>0, Q_{2}>0, U_{i}>0, S_{i}>0, W_{i}>0$ $(i=1,2 ; j=1,2)$, and any matrices $Z_{l}(l=1,2,3,4)$ of appropriate dimensions such that the LMIs (25)-(26) hold with $Q_{1}=0$.

Proof. Eliminating the term with $Q_{1}$ in LKF Equation (27) and proceeding the similar way as Theorem 1 yield Corollary 1. As the deriving process is directly following Theorem 1, it is omitted for the sake of simplicity.

Remark 6. When $d_{1}=0$, the interval $\left[0, d_{1}\right]$ is missing. It yields different selection of the LKF from the one in Theorem 1, as the $d_{1}$ in integral limit in Equation (27) becomes zero. Thus, it is more reasonable to reevaluate the Theorem 1 for $d_{1}=0$ and the following corollary is derived.

Corollary 2. Given scalars $d_{2}>d_{1}=0$ and $\mu>0$, the system (1) with a time delay $d(t)$ subject to (2) is asymptotically stable if there exist matrices $\bar{P}=\left[\bar{P}_{i j}\right]_{2 \times 2}>0, \bar{Q}_{i}>0, \bar{W}_{i}>0(i=1,2 ; j=1,2), \bar{U}>0$ and any matrices $\bar{Z}_{l}(l=1,2,3,4)$ of appropriate dimensions such that the following LMIs are feasible:

$$
\begin{gathered}
{\left[\begin{array}{cc}
\bar{U}+\bar{W}_{1} & \bar{Z}_{l} \\
* & \bar{U}+\bar{W}_{2}
\end{array}\right]>0(l=1,2,3,4),} \\
\bar{\Pi}_{i}=\operatorname{sym}\left\{\bar{\Omega}_{i}^{T} \overline{P \Lambda}\right\}+\bar{\Phi}_{1}+\bar{\Phi}_{2}+\bar{\Gamma}+\sum_{l=1}^{4} \bar{\Theta}_{l}<0(i=1,2),
\end{gathered}
$$

where

$$
\begin{gathered}
\bar{\Omega}_{1}=\left[\begin{array}{c}
e_{1} \\
d_{2} e_{5}
\end{array}\right], \bar{\Omega}_{2}=\left[\begin{array}{c}
e_{1} \\
d_{2} e_{4}
\end{array}\right], \bar{\Phi}_{1}=\left[\begin{array}{l}
e_{1} \\
e_{3}
\end{array}\right]^{T}\left[\begin{array}{cc}
\bar{Q}_{1} & 0 \\
* & -(1-\mu) \bar{Q}_{1}
\end{array}\right]\left[\begin{array}{l}
e_{1} \\
e_{3}
\end{array}\right]+\left[\begin{array}{l}
e_{1} \\
e_{2}
\end{array}\right]^{T}\left[\begin{array}{cc}
\bar{Q}_{2} & 0 \\
* & -\bar{Q}_{2}
\end{array}\right]\left[\begin{array}{l}
e_{1} \\
e_{2}
\end{array}\right], \\
\bar{\Lambda}=\left[\begin{array}{c}
A e_{1}+B e_{3} \\
e_{1}-e_{2}
\end{array}\right], \bar{\Phi}_{2}=\left(A e_{1}+B e_{3}\right)^{T}\left(d_{2}^{2} \bar{U}+\frac{d_{2}^{2}}{2}\left(\bar{W}_{1}+\bar{W}_{2}\right)\right)\left(A e_{1}+B e_{3}\right), \\
\widetilde{\Gamma}=\operatorname{col}\left\{\bar{\Gamma}_{3}, \bar{\Gamma}_{4}, \bar{\Gamma}_{5}, \bar{\Gamma}_{6}\right\}, \bar{\Gamma}=-\widetilde{\Gamma}^{T} \operatorname{diag}\left\{\varphi_{2}\left(\bar{W}_{1}\right), \varphi_{2}\left(\bar{W}_{1}\right), \varphi_{2}\left(\bar{W}_{2}\right), \varphi_{2}\left(\bar{W}_{2}\right)\right\} \widetilde{\Gamma} \\
\bar{\Gamma}_{1}=\operatorname{col}\left\{e_{1}-e_{3}, e_{1}+e_{3}-2 e_{4}, e_{1}-e_{3}+6 e_{4}-12 e_{6}, e_{1}+e_{3}-12 e_{4}+60 e_{6}-120 e_{8}\right\} \\
\bar{\Gamma}_{2}=\operatorname{col}\left\{e_{3}-e_{2}, e_{3}+e_{2}-2 e_{5}, e_{3}-e_{2}+6 e_{5}-12 e_{7}, e_{3}+e_{2}-12 e_{5}+60 e_{7}-120 e_{9}\right\}, \\
\bar{\Gamma}_{3}=\operatorname{col}\left\{e_{1}-e_{4}, e_{1}+2 e_{4}-6 e_{6}, e_{1}-3 e_{4}+24 e_{6}-60 e_{8}\right\} \\
\bar{\Gamma}_{4}=\operatorname{col}\left\{e_{3}-e_{5}, e_{3}+2 e_{5}-6 e_{7}, e_{3}-3 e_{5}+24 e_{7}-60 e_{9}\right\} \\
\bar{\Gamma}_{5}=\operatorname{col}\left\{e_{3}-e_{4}, e_{3}-4 e_{4}+6 e_{6}, e_{3}-9 e_{4}+36 e_{6}-60 e_{8}\right\} \\
\bar{\Gamma}_{6}=\operatorname{col}\left\{e_{2}-e_{5}, e_{2}-4 e_{5}+6 e_{7}, e_{2}-9 e_{5}+36 e_{7}-60 e_{9}\right\}, \\
\bar{\Theta}_{l}=-(2 l-1)\left[\begin{array}{c}
e_{1} \bar{\Gamma}_{1} \\
e_{1} \bar{\Gamma}_{2}
\end{array}\right]^{T}\left[\begin{array}{cc}
\bar{U} & \bar{Z}_{l} \\
* & \bar{U}
\end{array}\right]\left[\begin{array}{l}
e_{1} \bar{\Gamma}_{1} \\
e_{l} \bar{\Gamma}_{2}
\end{array}\right](l=1,2,3,4) .
\end{gathered}
$$

Proof. Choose the following LKF candidate

$$
\bar{V}(t)=\bar{V}_{1}(t)+\bar{V}_{2}(t)+\bar{V}_{3}(t)+\bar{V}_{4}(t),
$$


where

$$
\begin{aligned}
& \bar{V}_{1}(t)=\left[\begin{array}{c}
x(t) \\
\int_{t-d_{2}}^{t} x(s) d s
\end{array}\right]^{T}\left[\begin{array}{cc}
\bar{P}_{11} & \bar{P}_{12} \\
* & \bar{P}_{22}
\end{array}\right]\left[\begin{array}{c}
x(t) \\
\int_{t-d_{2}}^{t} x(s) d s
\end{array}\right], \\
& \bar{V}_{2}(t)=\int_{t-d(t)}^{t} x^{T}(s) \bar{Q}_{1} x(s) d s+\int_{t-d_{2}}^{t} x^{T}(s) \bar{Q}_{2} x(s) d s, \\
& \bar{V}_{3}(t)=d_{2} \int_{t-d_{2}}^{t} \int_{\theta}^{t} \dot{x}^{T}(s) \bar{U} \dot{x}(s) d s d \theta, \\
& \bar{V}_{4}(t)=\int_{t-d_{2}}^{t} \int_{\sigma}^{t} \int_{\theta}^{t} \dot{x}^{T}(s) \bar{W}_{1} \dot{x}(s) d s d \theta d \sigma+\int_{t-d_{2}}^{t} \int_{t-d_{2}}^{\sigma} \int_{\theta}^{t} \dot{x}^{T}(s) \bar{W}_{2} \dot{x}(s) d s d \theta d \sigma .
\end{aligned}
$$

The proof follows a similar fashion as that of Theorem 1 , which ultimately leads to

$$
\dot{\bar{V}}(t) \leq \bar{v}^{T}(t)\left(\frac{d_{2}-d(t)}{d_{2}} \bar{\Pi}_{1}+\frac{d(t)}{d_{2}} \bar{\Pi}_{2}\right) \bar{v}(t),
$$

where

$$
\begin{aligned}
& \bar{v}(t)=\operatorname{col}\left\{x(t), x\left(t-d_{2}\right), x(t-d(t)), \bar{c}_{1}, \cdots, \bar{c}_{6}\right\}, \bar{c}_{1}=\frac{1}{d(t)} \int_{t-d(t)}^{t} x(s) d s, \bar{c}_{2}=\frac{1}{d_{2}-d(t)} \int_{t-d_{2}}^{t-d(t)} x(s) d s, \\
& \bar{c}_{3}=\frac{1}{(d(t))^{2}} \int_{t-d(t)}^{t} \int_{\theta}^{t} x(s) d s d \theta, \bar{c}_{4}=\frac{1}{\left(d_{2}-d(t)\right)^{2}} \int_{t-d_{2}}^{t-d(t)} \int_{\theta}^{t-d(t)} x(s) d s d \theta, \bar{c}_{5}=\frac{1}{(d(t))^{3}} \int_{t-d(t)}^{t} \int_{\sigma}^{t} \int_{\theta}^{t} x(s) d s d \theta d \sigma, \\
& \bar{c}_{6}=\frac{1}{\left(d_{2}-d(t)\right)^{3}} \int_{t-d_{2}}^{t-d(t)} \int_{\sigma}^{t-d(t)} \int_{\theta}^{t-d(t)} x(s) d s d \theta d \sigma .
\end{aligned}
$$

Thus, if Equations (41)-(42) hold, the system (1) subject to (2) with $d_{1}=0$ is asymptotically stable. This ends the proof.

Remark 7. Besides the number of decision variables, the dimension of the LMI-based condition is also considered as a key factor for computational complexity [29]. Compared to $\Pi_{i}(i=1,2)$ in Theorem 1 , the dimensions of $\bar{\Pi}_{i}(i=1,2)$ in Corollary 2 are reduced from $13 n$ to $9 n$. Thus, Corollary 2 is more reasonable than Theorem 1 for systems with zero lower bounds.

\section{Numerical Examples}

In this section, three numerical examples are given to verify the improvement of the proposed approaches in reduction of conservatism. The conservatism is measured by the maximum allowable delay bounds (MADBs).

Example 1. Consider the following system with time delay:

$$
\dot{x}(t)=\left[\begin{array}{cc}
-2 & 0 \\
0 & -0.9
\end{array}\right] x(t)+\left[\begin{array}{cc}
-1 & 0 \\
-1 & -1
\end{array}\right] x(t-d(t))
$$

Case 1: For $\mu=0.9$ and given lower bounds of delay, the MADBs guaranteeing the stability of system (45) are listed in Table 1.

Table 1. MADBs $d_{2}$ for various $d_{1}$ and $\mu=0.9$ (Example 1 ).

\begin{tabular}{cccccc}
\hline Methods & $\boldsymbol{d}_{\mathbf{1}}=\mathbf{2}$ & $\boldsymbol{d}_{\mathbf{1}}=\mathbf{3}$ & $\boldsymbol{d}_{\mathbf{1}}=\mathbf{4}$ & $\boldsymbol{d}_{\mathbf{1}}=\mathbf{5}$ & Number of Variables \\
\hline Shao [30] & 2.5048 & 3.2591 & 4.0744 & - & $3 n^{2}+3 n$ \\
Sun et al. [14] & 2.5663 & 3.3408 & 4.1690 & 5.0275 & $18 n^{2}+8 n$ \\
Jiang and Han [31] & 2.5213 & 3.3311 & 4.1880 & 5.0722 & $6 n^{2}+4 n$ \\
Fridman et al. [32] & 2.7241 & 3.4580 & 4.2576 & 5.0977 & $11.5 n^{2}+3.5 n$ \\
Mohajerpoor et al. [25] & 2.9525 & 3.5837 & 4.3490 & 5.1623 & $25 n^{2}+5.5 n$ \\
Liu [33] & 3.0103 & 3.4865 & 4.1641 & 5.1012 & $17 n^{2}+8 n$ \\
Hien and Trinh [17] & 3.1634 & 3.6648 & 4.4467 & 5.2147 & $7 n^{2}+5 n$ \\
Theorem 1 & 3.9038 & 4.2741 & 4.8907 & 5.6759 & $12 n^{2}+6 n$ \\
\hline
\end{tabular}


The common feature of the methods in $[14,30,31]$ is the use of Jensen inequality for the estimation task, which introduces some conservatism. In [17], a new integral inequality is developed for single integral functional, which cannot be proved to include the Wirtinger inequality theoretically but could make use of more information on triple integrals of state, while in Theorem 1, the improved integral inequalities are utilized, through which more accurate bounds and more system information are obtained. From Table 1, one can see clearly that Theorem 1 leads to more desirable performance than the approaches in $[14,17,25,30-33]$ and involves fewer decision variables than those in $[14,33]$, which demonstrate the potential of the proposed integral inequalities in reduction of conservatism.

Case 2: When the derivative of the delay $\mu$ is unavailable, for different values of $d_{1}$, the comparisons of Corollary 1 with some recent ones are listed in Table 2.

Table 2. Maximum allowable delay bounds (MADBs) $d_{2}$ for various $d_{1}$ and unknown $\mu$ (Example 1).

\begin{tabular}{ccccccc}
\hline Methods & $\boldsymbol{d}_{\mathbf{1}}=\mathbf{1}$ & $\boldsymbol{d}_{\mathbf{1}}=\mathbf{2}$ & $\boldsymbol{d}_{\mathbf{1}}=\mathbf{3}$ & $\boldsymbol{d}_{\mathbf{1}}=\mathbf{4}$ & $\boldsymbol{d}_{\mathbf{1}}=\mathbf{5}$ & Number of Variables \\
\hline Shao [30] & 1.87 & 2.50 & 3.25 & 4.07 & - & $2.5 n^{2}+2.5 n$ \\
Zhu et al. [34] & 2.02 & 2.59 & 3.30 & 4.08 & - & $2.5 n^{2}+2.5 n$ \\
Tang et al. [35] & 2.04 & 2.60 & 3.30 & 4.08 & - & $2.5 n^{2}+2.5 n$ \\
Park et al. [27] & 2.06 & 2.61 & 3.31 & 4.09 & - & $3.5 n^{2}+3.5 n$ \\
Qian et al. [11] & 2.09 & 2.67 & 3.38 & 4.16 & - & $27.5 n^{2}+5.5 n$ \\
Fridman et al. [32] & 2.12 & 2.72 & 3.45 & 4.25 & 5.09 & $11.5 n^{2}+3.5 n$ \\
Hien and Trinh [23] & 2.31 & 2.80 & 3.50 & 4.30 & 5.14 & $19.5 n^{2}+4.5 n$ \\
Corollary 1 & 2.96 & 3.75 & 4.19 & 4.73 & 5.51 & $11.5 n^{2}+5.5 n$ \\
\hline
\end{tabular}

From Table 2, the methods in $[27,30,34,35]$ are inapplicable for $d_{1}=5$, since the used LKF terms contain no ' $t-d_{1}$ ' in the integral upper limit. Thus, the information on lower bound of delay is not used adequately. In Corollary 1, the quadratic functions in single, double and triple integral forms including ' $t-d_{1}$ ' are introduced into LKF. Moreover, $\int_{t-d_{1}}^{t} \int_{\theta}^{t} x(s) d s d \theta$ and $\int_{t-d_{1}}^{t} \int_{\sigma}^{t} \int_{\theta}^{t} x(s) d s d \theta d \sigma$ are utilized to form the augmented vector. As a result, Corollary 1 achieves improvements, especially for the larger lower bounds cases. In [11], the cross term in derivative of triple integral is converted to a quadratic function multiplied by a scalar and the relations among the state, delayed state and single integral of state are established by applying slack matrices. By Lemma 3, more relations among the double and triple integrals are obtained in the criterion without requiring extra slack variables. As seen from Table 2, compared to some existing ones including the refined Jensen inequality [23], Corollary 1 results in larger feasible stability regions with fewer variables than $[11,23]$. This verifies that the developed integral inequalities and construction of LKF are effective in deriving less conservative criterion.

Case 3: For the system with constant delay $\bar{d}$, the MADBs are calculated using the methods in $[16,19-21,36]$ and Corollary 1 (setting $d_{1}=d_{2}=\bar{d}$ ) are listed in Table 3.

Table 3. MADB for constant delay system (Example 1).

\begin{tabular}{ccc}
\hline Methods & $\overline{\boldsymbol{d}}$ & Number of Variables \\
\hline Kim [16] & 4.975 & $49 n^{2}+5 n$ \\
Zeng et al. [36] & 6.059 & $54 n^{2}+9 n$ \\
Seuret and Gouaisbaut [19] & 6.059 & $10 n^{2}+3 n$ \\
Zhang et al. [21] & 6.165 & $23 n^{2}+4 n$ \\
Zeng et al. [20] & 6.166 & $17.5 n^{2}+2.5 n$ \\
Corollary 1 & 6.170 & $11.5 n^{2}+5.5 n$ \\
\hline
\end{tabular}

In [36], a free-matrix-based integral inequality is developed that includes the Wirtinger inequality as a special case. Moreover, this approach has been improved in [20] by employing extra information on double integral of state. However, as discussed in [21], many slack variables are required in [20,36], bringing heavy computational burden. For two single integral terms with time delay, a novel 
integral inequality is proposed in [21], which provides smaller bounding gap than the combination of auxiliary function-based inequality and reciprocally convex lemma. Considering the constant delay case, the approach in [21] will reduce to the auxiliary function-based one. However, the estimation gaps of those inequalities have been further reduced in the Lemmas 2 and 3, which are independent on slack matrices. As seen from Table 3, Corollary 1 delivers better result and fewer variables than some recent integral inequality approach.

Example 2. Consider the following time-delay system:

$$
\dot{x}(t)=\left[\begin{array}{cc}
0 & 1 \\
-1 & -2
\end{array}\right] x(t)+\left[\begin{array}{cc}
0 & 0 \\
-1 & 1
\end{array}\right] x(t-d(t))
$$

Case 1: For $d_{1}=0$, the MADBs for various $\mu$ are presented in Table 4 .

From Table 4, it can be found that Corollary 2 outperforms some existing ones including delay decomposition [13], triple-integral term [14] and convex combination with delay partitioning [34,35]. It is noted that the LKFs in $[34,35]$ do not contain any triple integrals and their derivatives are estimated by Jensen inequality. In [13], the lower bound of delay is divided into two uniform segments to reduce the conservatism by larger lower bounds, while the computational burden will increase. In Corollary 2 , the same aim is achieved by augmented vector including information on the lower bound of delay, which yields less conservatism and fewer variables than [13].

Table 4. MADBs $d_{2}$ for various $\mu$ and $d_{1}=0$ (Example 2).

\begin{tabular}{cccccc}
\hline Methods & $\boldsymbol{\mu}=\mathbf{0 . 3}$ & $\boldsymbol{\mu}=\mathbf{0 . 5}$ & $\boldsymbol{\mu}=\mathbf{0 . 8}$ & $\boldsymbol{\mu}=\mathbf{1}$ & Number of Variables \\
\hline Shao [30] & 2.2160 & 1.1270 & 0.8710 & 0.8710 & $3 n^{2}+3 n$ \\
Sun et al. [14] & 2.2160 & 1.1272 & 0.8714 & 0.8714 & $18 n^{2}+8 n$ \\
Zhu et al. [34] & 2.2850 & 1.2080 & 1.0200 & 1.0200 & $3 n^{2}+3 n$ \\
Tang et al. [35] & 2.3070 & 1.2330 & 1.0440 & 1.0440 & $3 n^{2}+3 n$ \\
An et al. [13] & 3.6962 & 1.7655 & 1.3123 & 1.3123 & $25 n^{2}+9 n$ \\
Corollary 2 & 3.9872 & 2.0362 & 1.5110 & 1.4849 & $8.5 n^{2}+3.5 n$ \\
\hline
\end{tabular}

Case 2: When $\mu=0.3$, Table 5 gives the comparative results of the MADBs for different $d_{1}$.

Different from $[11,31]$, as discussed in Remark 4 , the relation between time delay and delay range is fully exploited in Theorem 1, which benefits the reduction of conservatism. This may explain that the methods of $[11,31]$ are more conservative than Theorem 1 in spite of having some triple-integral terms. Moreover, the Wiringer-based inequality [19] and new integral inequality [17] are encompassed as particular cases of that in Lemma 2 . From Table 5 , the results by Theorem 1 outperform those by $[14,15,17,19,37]$.

Table 5. MADBs $d_{2}$ for various $d_{1}$ and $\mu=0.3$ (Example 2).

\begin{tabular}{ccccc}
\hline Methods & $\boldsymbol{d}_{\mathbf{1}}=\mathbf{0 . 3}$ & $\boldsymbol{d}_{\mathbf{1}}=\mathbf{0 . 5}$ & $\boldsymbol{d}_{\mathbf{1}}=\mathbf{0 . 8}$ & $\boldsymbol{d}_{\mathbf{1}}=\mathbf{1}$ \\
\hline He et al. [37] & 2.190 & 2.200 & 2.200 & 2.210 \\
Sun et al. [14] & 2.263 & 2.285 & 2.307 & 2.316 \\
Feng et al. [15] & 2.432 & 2.433 & 2.430 & 2.423 \\
Seuret and Gouaisbaut [19] & 2.570 & 2.572 & 2.576 & 2.579 \\
Hien and Trinh [17] & 2.704 & 2.754 & 2.795 & 2.806 \\
Theorem 1 & 2.901 & 2.973 & 3.185 & 3.247 \\
\hline
\end{tabular}

Example 3. Consider the interval time-delay system:

$$
\dot{x}(t)=\left[\begin{array}{cc}
0 & 1 \\
-100 & -1
\end{array}\right] x(t)+\left[\begin{array}{cc}
0 & 0.1 \\
0.1 & 0.2
\end{array}\right] x(t-d(t))
$$


This example is investigated specifically for comparison with the Auxiliary-function based integral inequalities [26]. For the unknown $\mu$ and given $d_{1}$, the MADBs by the approach in [26] and Corollary 1 are listed in Table 6 . As pointed out in Remarks 1 and 2, the newly proposed inequalities have been proved to offer tighter bounds for both single and double integrals and make use of more system information. As a result, from Table 6, Corollary 1 achieves less conservatism and fewer variables than the method in [26], which demonstrate the advantages of Lemmas 2 and 3.

Table 6. MADBs $d_{2}$ for various $d_{1}$ and unknown $\mu$ (Example 3).

\begin{tabular}{cccccc}
\hline Methods & $\boldsymbol{d}_{\mathbf{1}}=\mathbf{0 . 1}$ & $\boldsymbol{d}_{\mathbf{1}}=\mathbf{0 . 2}$ & $\boldsymbol{d}_{\mathbf{1}}=\mathbf{0 . 3}$ & $\boldsymbol{d}_{\mathbf{1}}=\mathbf{0 . 4}$ & Number of Variables \\
\hline Park et al. [26] & 0.11 & 0.22 & 0.50 & 0.58 & $21 n^{2}+6 n$ \\
Corollary 1 & 0.19 & 0.28 & 0.51 & 0.61 & $11.5 n^{2}+5.5 n$ \\
\hline
\end{tabular}

\section{Conclusion}

In this paper, a series of novel integral inequalities for both single and double integrals is developed. Compared to the recently proposed inequalities, the obtained ones could provide more accurate estimations and utilize more system information without slack variables. Based on the integral inequalities, less conservative stability criteria for interval time-delay systems is obtained by constructing an augmented LKF. The information on the lower bounds of delay and the relationship between delay and delay interval are fully exploited. The numerical examples demonstrate the effectiveness of the proposed approaches in the reduction of conservatism.

Acknowledgments: The work is supported by the Fundamental Research Funds for the Central Universities under Grant 2014XS46.

Author Contributions: Both Shuai Zhang and Xiao Qi participated in the concept, design, analysis, and calculation. Xiao Qi wrote the draft and Shuai Zhang checked the manuscript. All authors read and approved the manuscript.

Conflicts of Interest: The authors declare no conflict of interest.

\section{References}

1. Guo, G.; Ding, L.; Han, Q.L. A distributed event-triggered transmission strategy for sampled-data consensus of multi-agent systems. Automatica 2014, 50, 1489-1496. [CrossRef]

2. Ding, L.; Han, Q.L.; Guo, G. Network-based leader-following consensus for distributed multi-agent systems. Automatica 2013, 49, 2281-2286. [CrossRef]

3. Li, H.Y.; Liu, H.H.; Gao, H.J.; Shi, P. Reliable fuzzy control for active suspension systems with actuator delay and fault. IEEE Trans. Fuzzy Syst. 2012, 20, 342-357. [CrossRef]

4. Gao, H.J.; Sun, W.C.; Shi, P. Robust sampled-data control for vehicle active suspension systems. IEEE Trans. Control Syst. Technol. 2010, 18, 238-245. [CrossRef]

5. Shao, H.Y.; Han, Q.L. New stability criteria for linear discrete-time systems with interval-like time-varying delays. IEEE Trans. Autom. Control 2011, 56, 619-625. [CrossRef]

6. Zhang, L.X.; Xiang, W.M. Mode-identifying time estimation and switching-delay tolerant control for switched systems: An elementary time unit approach. Automatica 2016, 64, 174-181. [CrossRef]

7. Zhou, B.; Egorov, A.V. Razumikhin and Krasovskii stability theorems for time-varying time-delay systems. Automatica 2016, 71, 281-291. [CrossRef]

8. Tian, Z.D.; Li, S.J.; Wang, Y.H.; Yu, H.X. Networked control system time-delay compensation based on time-delay prediction and improved implicit GPC. Algorithms 2015, 8, 3-18. [CrossRef]

9. Liu, Y.; Tao, T. A CS recovery algorithm for model and time delay identification of MISO-FIR systems. Algorithms 2015, 8, 743-753. [CrossRef]

10. Li, Z.C.; Bai, Y.; Huang, C.Z.; Yan, H.C. Further results on stabilization for interval time-delay systems via new integral inequality approach. ISA Trans. 2017, 68, 170-180. [CrossRef] [PubMed]

11. Qian, W.; Li, T.; Cong, S.; Fei, S.M. Stability analysis for interval time-varying delay systems based on time-varying bound integral method. J. Franklin Inst. 2014, 351, 4892-4903. [CrossRef] 
12. Ge, C.; Hua, C.C.; Guan, X.P. New delay-dependent stability criteria for neural networks with time-varying delay using delay-decomposition approach. IEEE Trans. Neural Netw. Learn. Syst. 2014, 25, 1378-1383.

13. An, J.Y.; Li, Z.Y.; Wang, X.M. A novel approach to delay-fractional-dependent stability criterion for linear systems with interval delay. ISA Trans. 2014, 53, 210-219. [CrossRef] [PubMed]

14. Sun, J.; Liu, G.P.; Chen, J.; Rees, D. Improved delay-range-dependent stability criteria for linear systems with time-varying delays. Automatica 2010, 46, 466-470. [CrossRef]

15. Feng, Z.G.; Lam, J.; Yang, G.H. Optimal partitioning method for stability analysis of continuous/discrete delay systems. Int. J. Robust Nonlinear Control 2015, 25, 559-574. [CrossRef]

16. Kim, J.H. Note on stability of linear systems with time-varying delay. Automatica 2011, 47, $2118-2121$. [CrossRef]

17. Hien, L.V.; Trinh, H. An enhanced stability criterion for time-delay systems via a new bounding technique. J. Franklin Inst. 2015, 352, 4407-4422. [CrossRef]

18. Briat, C. Convergence and equivalence results for the Jensen's inequality-application to time-delay and sample-data systems. IEEE Trans. Autom. Control 2011, 57, 1660-1665. [CrossRef]

19. Seuret, A.; Gouaisbaut, F. Wirtinger-based integral inequality: Application to time-delay systems. Automatica 2013, 49, 2860-2866. [CrossRef]

20. Zeng, H.B.; He, Y.; Wu, M.; She, J.H. New results on stability analysis for systems with discrete distributed delay. Automatica 2015, 60, 189-192. [CrossRef]

21. Zhang, C.K.; He, Y.; Jiang, L.; Wu, M.; Zeng, H.B. Stability analysis of systems with time-varying delay via relaxed integral inequalities. Syst. Control Lett. 2016, 92, 52-61. [CrossRef]

22. Park, M.J.; Kwon, O.M.; Park, J.H.; Lee, S.M.; Cha, E.J. Stability of time-delay systems via Wirtinger-based double integral inequality. Automatica 2015, 55, 204-208. [CrossRef]

23. Hien, L.V.; Trinh, H. Refined Jensen-based inequality approach to stability analysis of time-delay systems. IET Control Theory Appl. 2015, 9, 2188-2194. [CrossRef]

24. Mohajerpoor, R.; Shanmugam, L.; Abdi, H.; Rakkiyappan, R.; Nahavandi, S.; Park, J.H. Improved delay-dependent stability criteria for neutral systems with mixed interval time-varying delays and nonlinear disturbances. J. Franklin Inst. 2017, 354, 1169-1194. [CrossRef]

25. Mohajerpoor, R.; Shanmugam, L.; Abdi, H.; Rakkiyappan, R.; Nahavandi, S.; Shi, P. New delay range-dependent stability criteria for interval time-varying delay systems via Wirtinger-based inequalities. Int. J. Robust Nonlinear Control 2017. [CrossRef]

26. Park, P.G.; Lee, W.I.; Lee, S.Y. Auxiliary function-based integral inequalities for quadratic functions and their applications to time-delay systems. J. Franklin Inst. 2015, 352, 1378-1396. [CrossRef]

27. Park, P.G.; Ko, J.W.; Jeong, C. Reciprocally convex approach to stability of systems with time-varying delays. Automatica 2011, 47, 235-238. [CrossRef]

28. Sun, J.; Chen, J. Stability analysis of static recurrent neural networks with interval time-varying delay. Appl. Math. Comput. 2013, 221, 111-120. [CrossRef]

29. Zhang, C.K.; He, Y.; Jiang, L.; Wu, M. Stability analysis for delayed neural networks considering both conservativeness and complexity. IEEE Trans. Neural Netw. Learn. Syst. 2016, 27, 1486-1501. [CrossRef] [PubMed]

30. Shao, H.Y. New delay-dependent stability criteria for systems with interval delay. Automatica 2009, 45, 744-749. [CrossRef]

31. Jiang, X.F.; Han, Q.L. New stability criteria for linear systems with interval time-varying delay. Automatica 2008, 44, 2680-2685. [CrossRef]

32. Fridman, E.; Shaked, U.; Liu, K. New conditions for delay-derivative dependent stability. Automatica 2009, 45, 2723-2727. [CrossRef]

33. Liu, P.L. Further results on delay-range-dependent stability with additive time-varying delay systems. ISA Trans. 2014, 53, 258-266. [CrossRef] [PubMed]

34. Zhu, X.L.; Wang, Y.L.; Yang, G.H. New stability criteria for continuous-time systems with interval time-varying delay. IET Control Theory Appl. 2010, 4, 1101-1107. [CrossRef]

35. Tang, M.; Wang, Y.W.; Wen, C.Y. Improved delay-range-dependent stability criteria for linear systems with interval time-varying delays. IET Control Theory Appl. 2012, 6, 868-873. [CrossRef]

36. Zeng, H.B.; He, Y.; Wu, M.; She, J.H. Free-matrix-based integral inequality for stability analysis of systems with time-varying delay. IEEE Trans. Autom. Control 2015, 60, 2768-2772. [CrossRef] 
37. He, Y.; Wang, Q.G.; Lin, C.; Wu, M. Delay-range-dependent stability for systems with time-varying delay. Automatica 2007, 43, 371-376. [CrossRef] 